\title{
The history of little things that changed our lives
}

\author{
Saffet Mutluer
}

Received: 22 June 2011 / Accepted: 22 June 2011

(C) Springer-Verlag 2011

Being the president of an organization such as International Society for Pediatric Neurosurgery (ISPN) was truly an honor and a thoroughly thrilling experience. This is the point where I had dreamt of reaching in my professional career and this is the position of my teachers who deserved wearing this poncho. I would also like to thank everyone who supported me during this presidency.

One of the last assignments of an ISPN president is the presidential address. During the first session of this congress, we have watched the history of pediatric neurosurgery with a keen interest. Now, I would like to tell you about a different history, regarding the little things that changed our lives and our points of views.

Actually, I will tell you what you already know in a chronological order.

In 1923, Edwin Hubble discovered the Andromeda Galaxy and the presence of other galaxies beyond it. Until that time, it was thought that the whole universe comprised of our Milky Way Galaxy. He discovered one more thing: that the universe is expanding because of the drifting of the spectrum in the color red.

According to Hubble's finding, Russian cosmologist and mathematician Alexander Friedmann and the Belgian physicist priest Georges Lemaitre founded a theory concerning the formation of the universe.

In the beginning, there was nothing. Some 3.8 billion years ago, one day!

Big Bang!

There are many arguments and many things to tell on Big Bang. If we fast forward, one of the galaxies that was formed in spacetime is our Milky Way.

S. Mutluer $(\bowtie)$

Pediatric Neurosurgery, Ege University,

Izmir, Turkey

e-mail: saffetmutluer@gmail.com
The sun, which is one of the thousands of stars in this galaxy, is 26,000 light years away from the center.

Some 4.5 billion years ago, our planet, which was a fireball back then, slowly cooled down, and the supercontinent that is called Pangea, broke up into smaller continents that exist today 65,000 years ago.

Two million years ago, the first mammals appeared. Two hundred thousand years ago, the hominids, that are genetically $99 \%$ similar to us, learned to use tools. Let us all watch a small fragment from Stanley Kubrick's "A Space Odyssey 2001", which is one of the most important movie in the world's history of films, listening along to the famous opus from Strauss.

In this poetically shot scene, the ape man learns how to use the animal bone as a tool, for the first time. While he is breaking the other bones in front of him with this bone, the bone he throws spinning to the air metamorphs into a spaceship. Today, the Hubble telescope symbolizes this spaceship, and it helps us see the boundaries of our universe. The primitive man's learning of how to use his hands and tools marks the milestone in increasing of our knowledge.

Of course, we did not reach the invention of the Hubble telescope with hominids' first use of the tools. The spread of information took a long while.

\section{Doubling time of the knowledge}

The doubling of the knowledge from Stone Age to Bronze Age took millenniums. Until reaching the last century, although there was an acceleration caused by discoveries and inventions, the increasing of the knowledge has been slow.

In the second part of the twentieth century, the main reasons for the fast spread of information are the computers 
and the progress in the communication technologies (Table 1).

In 1966, I started the faculty of medicine. In that period, the concept of a computer was beyond imagination and even a copy machine was nonexistent. We would copy our study notes with a manifold writer and smear ink all over ourselves.

During my 6 years as a medical student, therapeutic medicine was primitive by today's standards. We had no H2 blockers or proton pump inhibitors for treating ulcers, no calcium channel blockers or ACE inhibitors for high blood pressure, no statins for high cholesterol, no Prozac for depression, and no vaccines for hepatitis or Haemophilus influenzae. There were no bone marrow transplants, liver transplants, or heart transplants. Coronary bypass operations and coronary angioplasty did not exist [4].

There were no CAT scans or MRIs. Alzheimer's disease was considered an extremely rare disorder, described briefly in Harrison's Principles of Internal Medicine (4th edition, 1962) and not even mentioned in Cecil-Loeb's Textbook of Medicine (11th edition, 1963). AIDS did not exist, and sickle cell anemia was the only inherited disease that had been studied at a molecular level [4].

In 1972, when I graduated from the faculty of medicine, many things had changed. Perhaps, the greatest advancement in a human being's life, called the "Chip", had been invented, which is one of the small things that affected our lives and way of living, and its many uses had entered our daily lives.

In those days, electrical engineers were aware on the potential of digital electronics; however, they faced a big limitation known as the "Tyranny of Numbers." This was the metaphor that described the exponentially increasing number of components required to design improved circuits against the physical limitations derived from the number of components that could be assembled together. Both, Kilby at Texas Instruments, and Noyce at Fairchild Semiconductor, were working on a solution to this problem during 1958 and 1959 [14].

Instead of designing smaller components, they found the way to fabricate entire networks of discrete components in a single sequence by laying them into a single crystal (chip) of semiconductor material. Kilby used germanium, and Noyce used silicon. The invention of Jack Kilby and Robert Noyce, also known as "the chip", has been recognized as one of the most important innovations and significant achievements in the history of humankind. The importance of the chip in the spread of information lies in its contribution to the computer technology. As a result of this invention, Jack Kilby was awarded with the Nobel Price in Physics in 2000 [14].

In July 18, 1968, Gordon Moore and Robert Noyce founded Intel (INTegrated ELectronic Corporation). They started the mass production of the first microprocessor, the 4004. Today, Intel is the firm that produces the biggest number of computer microprocessors.

The creation of the microchip caused a huge improvement in computer science.

"Who invented the computer?" is not a question with a simple answer. The real answer is that many inventors contributed to the history of computers and that a computer is a complex piece of machinery made up of many parts, each of which can be considered a separate invention.

Today, the inventor of the computer, which understands $\mathrm{ABCs}$ and is programmable, is John Atanasoff. After the invention of it, Electronic Numerical Integrator And Computer (ENIAC) came to being, which comprises of vacuum tubes and which was used during the World War II. ENIAC was the first electronic computer. It was a digital computer which was used for solving numerous computing problems. ENIAC was first designed for the US Army's Ballistic Research Laboratory, but was first used in special calculations for the hydrogen bomb [7]. ENIAC was a machine that weighed $30 \mathrm{t}$, in an area of $63 \mathrm{~m}^{2}$. We cannot compare its processing speed and capacity with the most basic models of cell phones.

We all know the rest from the first PC to now; it is reduced to a pocket's size.

So how was medicine affected by all of these?

One of the most important changes is the invention of CT.

The word "tomography" is derived from the Greek tomos (slice) and graphein (to write). Computed tomography was originally known as the "EMI scan" as it was developed at a research branch of EMI, a company best known today for its music and recording business. It was later known as computed axial tomography (CAT or CT scan) [24]. The first commercial CT scanner was invented by Sir Godfrey Hounsfield in Hayes, United Kingdom at EMI Central Research Laboratories using X-rays [2, 9]. Hounsfield conceived his idea in 1967 [24]. The first EMI scanner was installed in Atkinson Morley Hospital in Wimbledon, England, and the first patient brain scan was done on October 1, 1971. The invention of CT scan brought along a Nobel Price in Physiology or Medicine to Hounsfield in 1979 [5]. The original 1971 prototype took 160 parallel readings through 180 angles, each $1^{\circ}$ apart, with each scan taking a little over $5 \mathrm{~min}$. The images from these scans took $2.5 \mathrm{~h}$ to be processed by algebraic reconstruction techniques on a large computer. The scanner had a single photomultiplier detector, and operated on the translate/ rotate principle.

Today, with a CT scan with 128 collimator, it is possible to make almost any scanning in a minute. From one cross section in 5 min to 500 cross sections in a minute. Besides this, the control unit, which was the size of a room, was 
Table 1 Graph shows doubling time of knowledge. In the second part of the twentieth century, the main reasons for the fast spread of information are the computers and the progress in the communication technologies

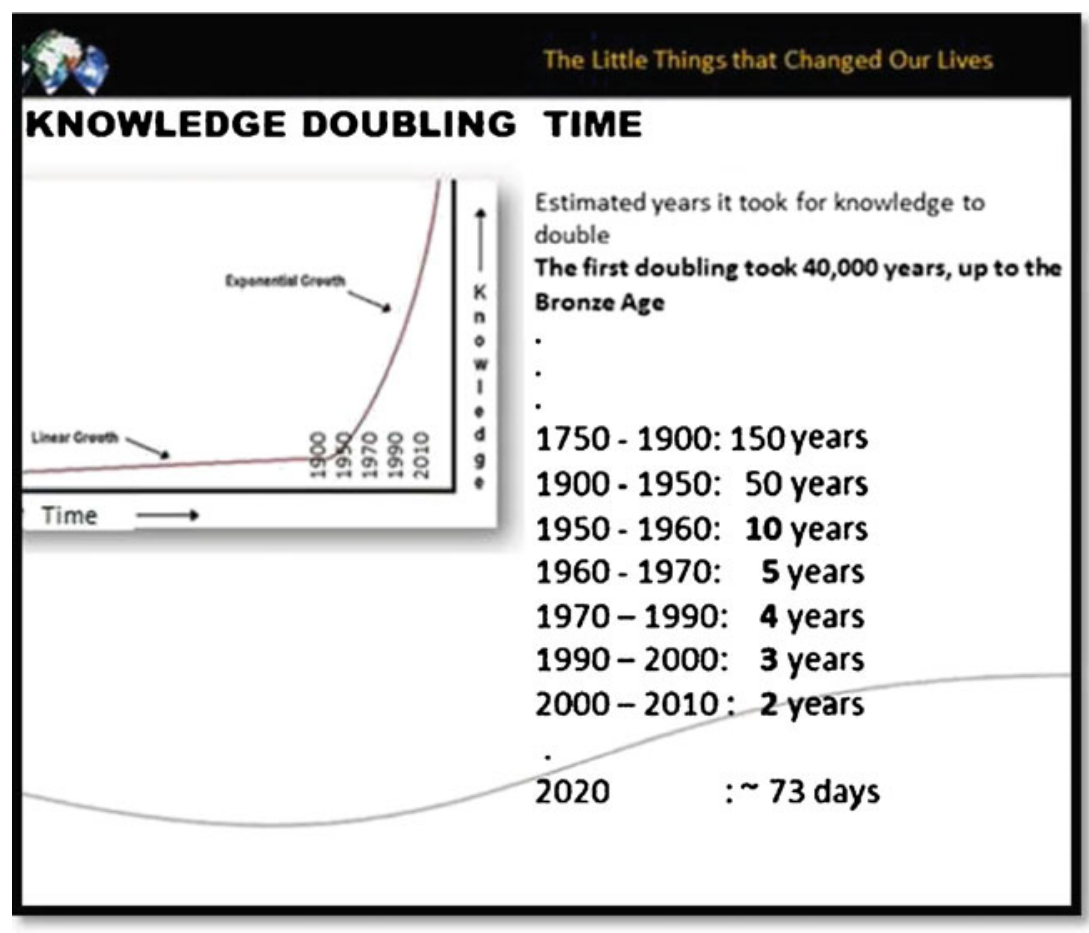

reduced to a keyboard and a screen on top of a desk. We can do all of this, thanks to this small thing called the "chip".

Magnetic resonance (MR) imaging is a relatively new technology. The first MR image was published in 1973, and the first cross-sectional image of a living mouse was published in January 1974. The first studies performed on humans were published in 1977 [16]. By comparison, the first human X-ray image was taken in 1895.

Today, by the use of MR, we do not only receive anatomical imaging. By using cine $\mathrm{MR}$, we can assess the brain functions through functional MR and we are able to preserve the eloquent areas. DTI shows the relation between the fiber direction and pathology. This helps us determine the approach to take without morbidity. In here, I am not going to mention ultrasonography, DSA, PET scan, and Gamma Knife, which are, as you all know, all computer-assisted methods.

The most important occurrence that affected the speed of the spread of information is the finding of the internet. It was first devised by Licklider from MIT in 1962. In 1965, through telephone cables between California and Massachusetts, two computers were connected. In 1969, ARPA net was founded. In 1972, the first e-mail and @ symbol came to being. In 1973, the first TCPI/IP Protocol were written. In 1986, the internet connections among universities were established. In 1989, folder transfer with $\mathrm{ftp}$ started. The year 1989 was the year for what these would eventually become the World Wide Web [7]. At CERN in Switzerland, Berners-Lee and Belgian computer scientist Robert Cailliau proposed in 1990 to use "HyperText
Transfer Protocol" to link and access information of kinds as a web of nodes in which the user can browse, and introduced the project in December to the public [23].

"The World Wide Web (W3) was developed to be a pool of human knowledge and human culture, which would allow collaborators in remote sites to share their ideas and all aspects of a common project" [23].

Today, thanks to www, the search engines, such as google, provide us with information in seconds.

ISPN also kept up with the times and did its part in the sharing of information by updating its website and by developing a "Guide". I would like to thank Rick Abbot, who had started this project.

Nowadays, many number of chips are in our houses, in our pockets, and on our arms. They are around the earth, perhaps in other planets, in outer space. If we were to ask the most important invention in the area of medicine as a result of the microchip and other discovery and inventions as a result of the microchip, I would say that it is the Hospital Integration System, which embodies all the aforementioned developments.

\section{Genes}

At this point, I would like to talk about another small thing which affects our lives profoundly: The Genes.

Before I began medical school, the gene had not yet become a household name. No one had ever isolated a gene or seen a gene. Genes could not be purified or put in a bottle. 
Only 3 of the 100 medical schools in the USA had postdoctoral specialty training in medical genetics [4].

The year that I began medical school, 1966, was a turning point in the history of genetics. This was the year that the genetic code was completely deciphered. Six years later when I graduated, in 1972, the technique of recombinant DNA was discovered.

By the early 1980 s, scientists had perfected the techniques to clone and sequence individual genes and to study their action in living cells of animals and humans. Today, genes can be cloned, sequenced, weighed, counted, visualized, replicated, altered in test tubes, and shuttled from cell to cell in animals and humans.

Today, manipulating genes is as easy as surfing the Web. Every week, the front page of every major newspaper reports the discovery of a new disease-producing gene or a potentially new gene-based drug. We may soon live in a world where the Golden Arches yield center stage to the Golden Helices [4].

"A gene is a unit of heredity in a living organism. A modern working definition of a gene is a locatable region of genomic sequence, corresponding to a unit of inheritance, which is associated with regulatory regions, transcribed regions, and or other functional sequence regions" [8].

If there is such a thing called fate, it is written on our genes. The current representation of numeric values in the binary system in numbers 1 and 0 is in octadecimal values in genes. The codons with combinations of A, G, C, T are the foundation blocks of this code (Table 2).

\section{Some important milestones in the development of medical genetics}

\section{Experiments on plant hybridization}

Gregor Mendel, who is known as the "father of modern genetics", was inspired by both his professors at the university and his colleagues at the monastery to study variation in plants. Between 1856 and 1863, Mendel cultivated and tested some 29,000 pea plants (i.e., Pisum sativum). This study showed that one in four pea plants had purebred recessive alleles, two out of four were hybrid and one out of four were purebred dominant. His experiments were later became known as Mendel's Laws of Inheritance [10].

In 1940, George Beadle and Edward Tatum demonstrated the existence of a precise relationship between genes and proteins [11]. In the course of their experiments connecting genetics with biochemistry, they switched from the genetics mainstay Drosophila to a more appropriate model organism, would become a recurring theme in the development of molecular biology. This discovery led Beadle and Tatum to win the 1958 Nobel Prize in Physiology and Medicine
Table 2 Computer coding and genetic coding

001101011010100010111101011010110100101011010111010 110001010100110101111010110110000110101101010110101 101010110101010110101111001110000110101011100101010 110010101010111101010110101110101101011101010100111 101001100101011101010111100101011101000010110101100 001101011010100010111101011010110100101011010111010 110001010100110101111010110110000110101101010110101 101010110101010110101111001110000110101011100101010 110010101010111101010110101110101101011101010100111 101001100101011101010111100101011101000010110101100 001101011010100010111101011010110100101011010111010 110001010100110101111010110110000110101101010110101 101010110101010110101111001110000110101011100101010 110010101010111101010110101110101101011101010100111

TGGCTGAT TCTTCTAG GGITIGGA GGTGGGA TCTTCAGC

TCTCTACG TCTCTTCG GGITIGGA GGTTGAGA TCTITAG

TGGCTGAT TCTTCTAG GGTITGGA GGTGGGAG TCTTCAGC

TCTCTACG TCTCTTCG GGTITGA GGTTGAGA TCTITTAG

TGGCTGAT TCTTCTAG GGITIGGA GGTGGGAG TCTTCAGC

TCTCTACG TCTCTICG GGTITGA GGTTGAGA TCTITIAG

TGGCTGAT TCTTCTAG GGTITGGA GGTGGGAG TCTTCAGC

TCTCTACG TCTCTTCG GGTITGGA GGTTGAGA TCTITIAG

TGGCTGAT TCTTCTAG GGTITGGA GGTGGGAG TCTTCAGC

TCTCTACG TCTCTTCG GGTITGGA GGTTGAGA TCTITIAG

TGGCTGAT TCTTCTAG GGTITGGA GGTGGGAG TCTTCAGC

TCTCTACG TCTCTTCG GGITIGGA GGTTGAGA TCTITIAG

TGGCTGAT TCTTCTAG GGTITGGA GGTGGGAG TCTTCAGC

TCTCTACG TCTCTICG GGTITGA GGTIGAGA TCTITIAG

[11]. In 1944, Oswald Avery, demonstrated that genes are made up of DNA

"Photo 51 " is the nickname given to an X-ray diffraction image of DNA taken by Rosalind Franklin in 1952 that was critical evidence in identifying the structure of DNA. Nature was the first publication of this more clarified Xray image of DNA. The " $\mathrm{X}$ " in the image comes from the zigzag profile of the helix [20]. In the light of this data, James Watson and James Crick discovered DNA's doublehelix configuration. This discovery caused them to be awarded with the 1962 Nobel Prize in Medicine [20].

In 1953, the fundamental question was: How does DNA inside the cell's nucleus?

Nonribosomal, short-living tRNAs were discovered. The genetic code was deciphered, and codons belonging to 20 amino acids were found. Marshal Nirenberg and Gobin Koharana were awarded the 1965 Nobel Prize in Medicine with this discovery [15].

For long years, it was thought that mRNAs were completely processed. In 1977, it was discovered that mRNAs were first synthesized as pre-RNAs and later became mature mRNAs (Sharp and Roberts won the 1993 Nobel Prize in Medicine).

In 1989, Carry Mullin found the polymerase chain reaction (PCR) as a scientific technique in molecular biology to amplify a single or a few copies of a piece of 
DNA across several orders of magnitude, generating thousands to millions of copies of a particular DNA sequence (Mullin was awarded with the 1993 Nobel Prize in Medicine due to this discovery) [19].

The Human Genome Project (HGP) is a scientific research project with the aim of determining the sequence of chemical base pairs which make up DNA and to determine and map the approximately 25,000 genes of the human genome both physically and functionally. The project began in 1990 and was first led by James D. Watson at the US National Institutes of Health. A draft of the genome project was released in 2000 and a complete one in 2003. Still, more detailed analyses are still being published [13].

Now, we have a large library. The sequence of human DNA is stored in database to anyone on the internet.

Fire and Mello could not acquire adequate mRNA silencing in the sense and antisense RNA in a cell. However, with a double-stranded RNA, the silencing was achieved. The target mRNA dissappeared after silencing. RNA interference: A powerful tool in the blocking of a gene's function. In medical practice, especially in treatment, new approaches (this discovery caused Fire and Mello to be awarded with the 2006 Nobel Prize in Medicine) [21].

Functional Annotation of Mouse. FANTOM association discovered different DNA transcripts, which does not have a translation and which has functions such as RNA molecules (Table 3 shows non-coding RNAs).

One of the most important non-coding RNAs is the MicroRNA (miRNA).

Today, more than 4,000 different miRNA in various organisms and more than 900 different miRNA in human beings have been identified.

As of 2010, there are more than 600 articles in PUBMED.

The functions of miRNA are:

- Immune response

- Organogenesis

Table 3 Some of the non-coding RNAs
1. Transfer RNA (tRNA)
2. Ribozomal RNA (rRNA)
3. Small nuclear RNA (snRNA)
4. Small nucleolar RNA (snoRNA)
5. Small cajal body-specific RNA (scaRNA)
6. microRNA (miRNA)
7. Guide RNA (gRNA)
8. Signal recognizing particle (SRP) RNA
9. Promotor RNA (pRNA)
10. Efference RNA (eRNA)
11. tmRNA

- Cellular differentiation, growth, and apoptosis

- Endocrine system

- Haematopoiesis

The new treatment strategy in genetics is "RNA interference". Besides the studies that show treatment efficiency in animals:

Alnylam, which is developed against RSV and NUC B1000, which is developed against HBV are in the first phase of clinical trials. The production is easy and cheap. The local applications are easy to do. Their toxicity is low, and they are very specified [5].

Like the microchip, which ushered a new era in electronics, I believe that microRNAs will usher a new era in medicine, and I also believe that new treatment methods will be discovered in the curing of tumors and infections by using RNA interference.

\section{Nanotechnology}

Microchip changed our lives in every field. Genetic studies are continuing to change. But recent 10-year production of nanoparticles and nanotechnology seems to change everything. Understanding of nanotechnology may be easier than to express in a short time.

Nano means dwarf in old greek.

Humans have, without knowing, used nanotechnology for thousands of years for such things as in making steel and in vulcanizing rubber [18]. Each of these rely on the properties of coincidentally formed atomic ensembles and are distinguished from chemistry in that they do not depend on the properties of individual molecules. But the development of concepts now included under the term nanotechnology has been slower.

The first mention of some of the distinguishing concepts in nanotechnology (but predating use of that name) was in 1867 by James Clerk Maxwell when he proposed as a thought experiment a tiny entity known as Maxwell's Demon able to handle individual molecules [2].

The first observations and size measurements of nanoparticles was made during the first decade of the twentieth century. They are mostly associated with Richard Adolf Zsigmondy who made a detailed study of gold sols and other nanomaterials with sizes down to $10 \mathrm{~nm}$ and less. He published a book in 1914. He used ultramicroscope that employs the darkfield method for seeing particles with sizes much less than light wavelength. Zsigmondy was also the first who used nanometer explicitly for characterizing particle size [2].

The topic of nanotechnology was again touched upon by "There's Plenty of Room at the Bottom," a talk given by physicist Richard Feynman at an American Physical 
Society meeting at Caltech on December 29, 1959 (Feynman, with his quantum mechanics studies, was awarded with the 1965 Nobel Prize in Physics) [2].

Feynman defined a process by which the ability to manipulate individual atoms and molecules might be put to use, using one set of exact tools to build and operate another proportionally smaller set. In this, he noted, scaling problems would arise from the altering of magnitude of various physical phenomena: gravity would be less important, surface tension and Van der Waals attraction would be more important. This basic idea appears feasible, producing a useful quantity of end products.

At this meeting, Feynman announced two problems, and he offered to pay a sum of $\$ 1,000$ for the first persons to solve each one. The first challenge was the construction of a nanomotor, which was achieved on November 1960 by William McLellan. The second one was the possibility of minimizing letters small enough so that they would be able to fit the entire Encyclopedia Britannica on the head of a pin, and this prize was won in 1985 by Tom Newman $[1,12]$.

A scanning tunneling microscope (STM) is a powerful instrument for imaging surfaces at the atomic level. Its development in 1981 earned its inventors, Gerd Binnig and Heinrich Rohrer (at IBM Zürich) the Nobel Prize in Physics in 1986 [20, 21]. For an STM, good resolution is considered to be $0.1 \mathrm{~nm}$ lateral resolution and $0.01 \mathrm{~nm}$ depth resolution. With this resolution, individual atoms within materials are routinely imaged and manipulated. The STM can be used not only in ultra-high vacuum but also in air, water, and various other liquid or gas ambients, and at temperatures ranging from near $0 \mathrm{~K}$ to a few hundred degrees Celsius [22].

What is nano-technology?

Nanotechnology, shortened to "nanotech", is the study of the controlling of matter on an atomic and molecular scale. Generally, nanotechnology deals with structures sized between 1 and $100 \mathrm{~nm}$ in at least one dimension, and involves developing materials or devices within that size [18].

Nanotechnology and nanoscience got started in the early 1980s with two major developments; the birth of cluster science and the invention of the STM. This development led to the discovery of fullerenes in 1985 and carbon nanotubes a few years later. In another development, the synthesis and properties of semiconductor nanocrystals was studied; this led to a fast increasing number of metal and metal oxide nanoparticles and quantum dots. The atomic force microscope (AFM or SFM) was invented 6 years after the STM was invented [6].

We are using many organic and non-organic materials to produce nanomaterials.
Nano materials:

- Nanoparticles

- Quantum dots

- Nanotubes

- Fullerenes

In our daly lives, we are using many nanotech production without knowing the technology underneath.

Nanomedicine is the medical application of nanotechnology [17]. Nanomedicine ranges from the medical applications of nanomaterials to nanoelectronic biosensors, and even possible future applications of molecular nanotechnology.

Quantum dots

By way of review, quantum dots (qdots) are nanometersized semiconductor nanocrystals, made of cadmium selenide (CdSe), cadmium sulfide (CdS), or cadmium telluride (CdTe), surrounded by a polymer coating that is inert. The core of the nanocrystal is chosen depending on the emission wavelength range that is being targeted: $\mathrm{CdS}$ for ultraviolet blue, CdSe for the majority of the visible spectrum [25].

With qdots, it is now possible to observe the activities inside the living cells and to look at them through a microscope. When the skin is exposed to ultraviolet rays, it is feasible to see the lymph canals with the help of the qdots which are traveling inside the lymph gland. Through this, it is possible to reach lymph nodes by small incisions [25].

\section{Magnetic nanoparticles}

Magnetic nanoparticles are becoming of utmost importance as tools in medical and biological diagnostics. They have become especially useful as contrast agents in magnetic resonance imaging (MRI) [25]. Superparamagnetic iron oxide nanoparticles (SPIOs), also known as monocrystalline iron oxide nanoparticles (MIONs), have an inorganic core of iron oxide surrounded by a polymer coating of dextran or polyethylenglycol (PEG) molecules. The iron oxide crystal core becomes magnetized when placed in an external magnetic field [25].

An ultrasmall superparamagnetic iron oxide nanoparticle (USPIO) was evaluated as an imaging agent in patients with malignant brain tumors both pre- and postoperatively [25] This viral-sized nanoparticle is known to show enhancement of malignant intracranial tumors. All patients had tumors that enhanced on first MRI scans. However, the most important thing was that, in several of the patients, there were areas that showed enhancement with the nanoparticle agent, but not with gadolinium [26]. 
The operative field

Currently, saline irrigation is the most commonly used procedure for clearing blood and debris from the operative field during brain or spine surgery. This time-consuming technique is often repeated throughout the surgery. In recent work, a self-assembling, peptide nanofiber scaffold was used to create a clear gel biological barrier that covered the operative field during brain surgery [3]. The self-assembling peptide nanofiber gel was applied as a clear liquid and formed a transparent gel that prevented movement of blood and debris into or out of the operative field. The surgery proceeded within this gel. Results using this nanofiber scaffold gel in rodent brain and spine surgery are promising.

Hemostasis during neurosurgical procedures typically involves the use of electrocautery, direct pressure, and procoagulation materials, as well as application of adhesive products such as fibrin glue. A newly reported method of hemostasis used a self-assembling peptide that was capable of achieving hemostasis in a wet ionic environment in less than $15 \mathrm{~s}$ [3].

\section{Central nervous system repair}

Focal damage to central nervous system (CNS) tissue may occur secondary to multiple causes including trauma, ischemic or hemorrhagic stroke, and neoplasm. Propagation of CNS damage may occur as a result of free-radical injury. Hydroxyl functionalized C-60 fullerene derivatives, called fullerenols, have demonstrated usefulness as neuroprotective agents [3]. These soccer ball-shaped nanoparticles possess antioxidant and free-radical-scavenger capabilities that decrease receptor-mediated excitotoxic and apoptotic cell death. This effect is likely due, in part, to inhibition of glutamate receptor bonding and reduction in glutamateinduced increases in intracellular calcium.

Restoration of lost function depends in part on axonal regeneration. CNS axonal regeneration has been demonstrated in Hamsters [3].

\section{Intraoperative endoscopy}

The use of the operative microscope more than a generation ago was a milestone event in the field of neurosurgery [2]. The detail and scale available in the operating theater basically changed ideas and approaches to neurological diseases.

The incorporation of nanotechnology into the practice of neurosurgeons may change, by orders of magnitude, the scale and complexity at which neurological diseases may be examined and remedied.

Micromirror chip technology became instrumental in confocal microscopy and endoscopy [2], and its use in neurosurgical operative endoscopes and microscopes can improve image quality.

Another challenge regarding endoscopy is the limited space available for instrumentation. The imaging apparatus, irrigation, and light source occupy most of the endoscope. Use of nanotechnology will decrease the scale of the functional ends of each of these and cause further minimalization of the procedure overall. New instruments can use nanosensors for in vivo real-time histopathology and genetic analysis of intracranial neoplasms [2]. The DMD can also be used for high-speed confocal microscopy to create three-dimensional images.

\section{Drug delivey}

New drug delivery technologies based on nanotechnology reflect an important objective of the pharmaceutical industry, and numerous applications are nowadays emerging.

These applications are made to improve controlled drug release, increase efficacy, improve patient safety and compliance, minimize side effects, and secure targetspecific drug release, among others [3]. Also, one of the basic advantages of nanoscale drug delivery vehicles is in their ability to penetrate membrane barriers, especially in the CNS and gastrointestinal tract.

\section{Polymeric nanoparticles}

Polymeric nanoparticles consist of colloidal particles that carry a drug of interest within a biodegradable polymer matrix. Depending on the method of preparation, nanoparticles, nanospheres, or nanocapsules can be produced. Nanospheres consist of a polymer matrix in which the drug is physically and uniformly dispersed, whereas nanocapsules represent vesicular carrier systems that confine the drug to a central cavity surrounded by a polymer matrix [3].

\section{Dendrimers}

Dendrimers are highly branched macromolecules with a controlled three-dimensional structure that surrounds a central core [3]. Polymer growth begins from a central core molecule and stretches outward through a series of polymerization reactions. So, the size of these dendritic nanocarriers can be precisely controlled in the range of 5 $20 \mathrm{~nm}$ [3]. Channels and cages can be created within gaps in the core structure where drug molecules or DNA strands can be attached. Because of their structure, one dendrimer is able to transport very high densities of drug molecules when compared with other nanoparticle-based carrier systems.

In January 2005, the United States Food and Drug Administration approved the use of Abraxane (Abraxis 
Oncology, Schaumburg, IL, USA) for treating patients with breast cancer resistant to conventional chemotherapy. Abraxane consists of a solvent-free, protein-stabilized nanoparticle formulation of paclitaxel [3]. Results of the phases II and III trials of Abraxane against conventional Taxol showed significantly higher response rates. Besides these, the nanoparticle formulation can be administered over $30 \mathrm{~min}$ once every 3 weeks without premedication, whereas Taxol (Bristol-Myers Squibb, Princeton, NJ, USA) requires $3 \mathrm{~h}$ to administer, and premedication with steroids and antihistamines is needed [3].

Moore's Law, which has held true for 40 years, states that the number of transistors on an integrated circuit doubles every 2 years while maintaining cost effectiveness [2]. This law effectively describes advancements in processing speed as a function of time and holds true if extended further back in time to the era of the vacuum tube. Moore's Law extended backward and forward over time. The inclusion of technologies, such as vacuum tubes, shows remarkable adherence to Moore's Law.

Nowadays, nanotechnology keeps sustaining the developments in microprocessors. With 2, 4, and even 8 core processors, the development according to Moore's Law is ongoing. Also, the data storage in discs has been converted into more data storage in smaller spaces.

In a decade, we will see that the nanotechnology is gaining more ground in the field of medicine in both diagnosis and treatment. Most of these will be reflected in our applications in a year or two.

Since the nineteenth century, once in 50 years, the revolutionary driving forces caused very important changes in our lives. Whether picotechnology will replace microtechnology and nanotechnology is yet to be seen, and perhaps, we will see this before the twenty first century ends.

My conclusion is: the things that changed our lives have always been small things. The people who dealt with these small things have been awarded with the Nobel Prize. However, to challenge to change our lives require knowledge, skills, art, and respect.

\section{References}

1. Adleman LM (1994) Molecular computation of solutions to combinatorial problems. Science 266:1021-1024

2. Elder JB, Liu CY, Apuzzo ML (2008) Neurosurgery in the realm of 10(-9), part 1: stardust and nanotechnology in neuroscience. Neurosurgery 62:1-20

3. Elder JB, Liu CY, Apuzzo ML (2008) Neurosurgery in the realm of $10(-9)$, Part 2: applications of nanotechnology to neurosurgerypresent and future. Neurosurgery 62:29-284

4. Goldstein JL (1999) Burgers, chips, and genes. Ann NY Acad Sci $882: 8-21$

5. http://en.wikipedia.org/wiki/Alnylam

6. http://en.wikipedia.org/wiki/Atomic_Force_Microscope

7. http://en.wikipedia.org/wiki/ENIAC

8. http://en.wikipedia.org/wiki/Gene

9. http://en.wikipedia.org/wiki/Godfrey Hounsfield

10. http://en.wikipedia.org/wiki/Gregor_mendel

11. http://en.wikipedia.org/wiki/History_of molecular_biology

12. http://en.wikipedia.org/wiki/History_of_nanotechnology

13. http://en.wikipedia.org/wiki/Human_Genom_Project

14. http://en.wikipedia.org/wiki/Integrated circuit

15. http://en.wikipedia.org/wiki/Marshall_Warren_Nirenberg

16. http://en.wikipedia.org/wiki/MRI

17. http://en.wikipedia.org/wiki/Nanomedicine

18. http://en.wikipedia.org/wiki/Nanotechnology\#cite_note-1

19. http://en.wikipedia.org/wiki/PCR

20. http://en.wikipedia.org/wiki/Photo_51

21. http://en.wikipedia.org/wiki/RNA_interference

22. http://en.wikipedia.org/wiki/Scanning_tunneling_microscope

23. http://en.wikipedia.org/wiki/www

24. http://en.wikipedia.org/wiki/X-ray_computed_tomography

25. Leary SP, Liu CY, Apuzzo ML (2006) Toward the emergence of nanoneurosurgery: part II-nanomedicine: diagnostics and imaging at the nanoscale level. Neurosurgery 58:805-823

26. Leary SP, Liu CY, Apuzzo ML (2006) Toward the emergence of nanoneurosurgery: part III-nanomedicine: targeted nanotherapy, nanosurgery, and progress toward the realization of nanoneurosurgery. Neurosurgery 58:1009-1026 